\title{
A Nail Box Machine Manipulator Design
}

\author{
Changzhong $\mathrm{Wu}^{\mathrm{a}}$, Xiuguo $\mathrm{Wei}^{\mathrm{b}}$, Mingqing $\mathrm{Wu}^{\mathrm{c}}$ \\ School of Mechanical Engineering, University of Jinan, Jinan250022, China \\ Shandong vocational college of transportation and technology, Weifang 261206, China \\ achangzhongwu@163.com, '530149460@qq.com, ${ }^{\mathrm{c}} 24240336 @ q q . c o m$
}

\begin{abstract}
In the present paper, we carry out the automatic modification of a semi-automatic nail case machine with a manipulator as the main body, in order to meet the needs of the market. In particular, the design of the main parts of the manipulator and the pneumatic system are described in detail. The designed manipulator can save the labor force, enhance the continuity and stability of production, and improve production efficiency compared to the traditional devices.
\end{abstract}

Keywords-Nail case machine, Automation, Pneumatic, Manipulator

\section{INTRODUCTION}

In the current, the main drawback of the automatic nailing machine is the high equipment costing, and the production line is too long, high requirement to the ground. The semi-automatic nail box machines have some negative factors, such as low production efficiency, high labor intensity etc.. ${ }^{[1]}$.

Based on the above situation, design of a miniature automatic nail box machine system, which basically have all valid function of the automatic nail box machine, automatic feeding, automatic bending, automatic feed and automatic nail ${ }^{[2]}$. Which greatly reduces the occupied area, it effectively saves the cost, and improves the degree of automation, and the labor intensity is greatly reduced, enhanced the continuity and stability of production, improved production efficiency. Compared with the existing automatic equipment it has the advantages of simple structure, low cost has a good popularization value.

\section{The Overall Design OF MANIPULATOR SyStem}

The main function unit of the manipulator system is designed shown in Fig. 1.

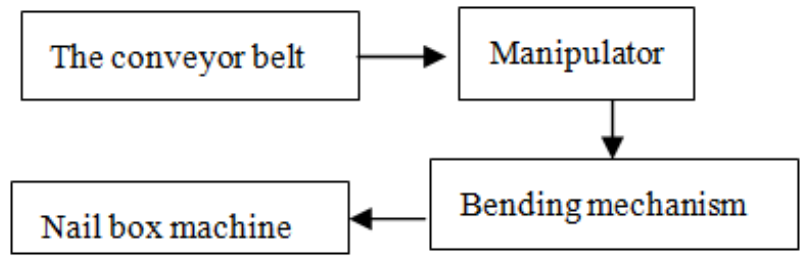

Fig. 1. Main function unit of the manipulator system

\section{A. Design of belt conveyor unit.}

The conveyor belt is the supply unit of board. In the designing, which require corrugated cardboard can be transported from the head of the conveyor belt to end with single time and continuity[3]. In the design which learn from the traditional conveyor belt conveyor, and set a vertical baffle front belt, so the operator can adjust the height $\mathrm{h}$ between the baffle and the work surface of conveyor belt, which need to satisfied $\mathrm{d}<\mathrm{h}<1.7 \mathrm{~d}$ ( $\mathrm{d}$ is the single corrugated cardboard thickness) by the sides of the locking bolt ${ }^{[4]}$. To adapt to the different width of the board, extending the length of the baffle which is on both sides of the conveyer belt is needed. A gantry type structure is designed above the transportation belt, from its beam hanging two flattening wheel, and adjusting the space of them to rolling in the two bending line.

The conveyor belt is designed in three sections, including 6 power roller, and a segment is composed of two power rollers, which length is designed length of 1.8 meters, with a three-phase asynchronous motor as a power source. They are connected by a chain and sprocket. Each section is designed with no power roller, and used of plastic roller having bearing as the supporter for conveyer belt. The design of the structure of conveying belt is shown in Fig. 2.

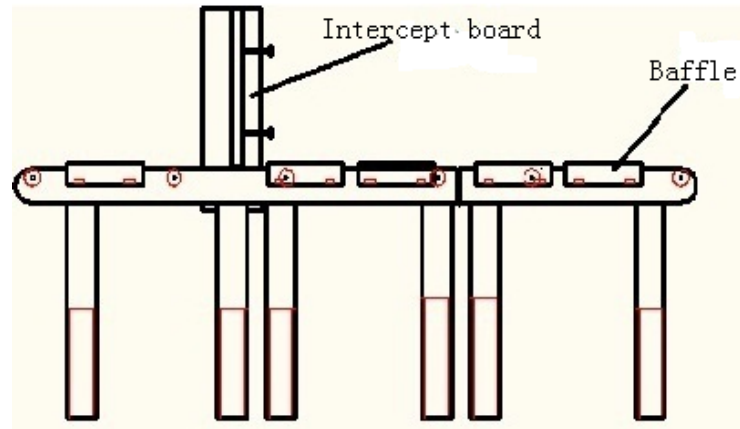

Fig. 2. Design of the structure of conveying belt

\section{B. The design of manipulator unit}

1) The structure and the characteristics of Manipulator

The pneumatic components as the execution are adopted in the design of Longman mechanical hands. According to the characteristics by size of the actual operation of the work piece (large plane), it is decided to adopt the design of double manipulator, and by adjusting the spacing in the light bar of the manipulator to meet machining requirements of different sizes of cardboard, making the force of the board balanced ${ }^{[5]}$, avoiding the cardboard bending which is due to the uneven force on the large cardboard surface, and effecting of the next step.

Considering the different size of cardboard, for the realization of the longitudinal, regulate the sucker's position in a board. The Longman type manipulator is designed into I-shaped frame structure, mounting gear rack and rails on both 
sides of the supporting seat, a three-phase asynchronous motor as a power source, through the gear transmission, to realize the purpose of manipulator with the beam longitudinal sliding. Because the mechanical hand's vertical activity frequency is low, and to reduce the complexity of program design, at the same time, to meet the adjusted longitudinal movement randomly. The motor power system is designed by independently jog control. The 3D model is shown in Fig. 3.

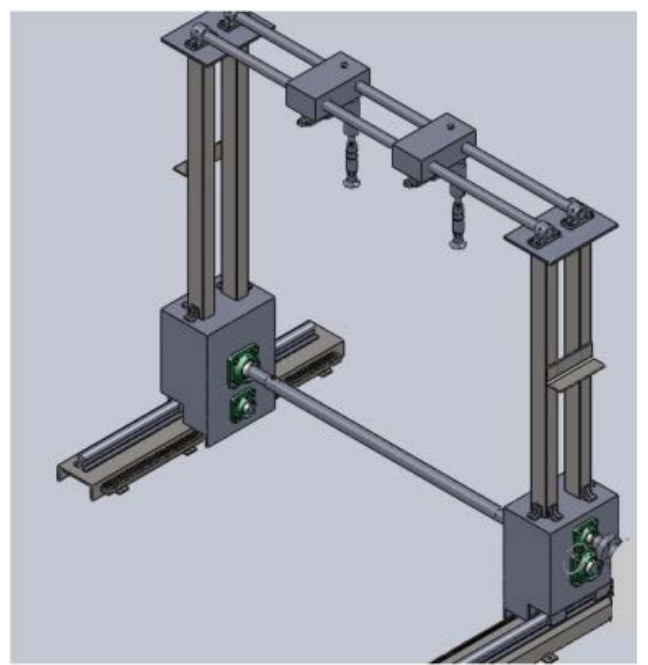

Fig. 3. 3D model

2) Design and calculation of main parts of the manipulator

(1)Longman frame structure is mainly as a mounting platform of the pneumatic manipulator, and the longitudinal motion accuracy requirement is low[6]. According to the requirements of the basic design parameters: the longitudinal moving rail length is $1200 \mathrm{~mm}$; mechanical arm medial lateral span is $1800 \mathrm{~mm}$; the longitudinal velocity is $240 \mathrm{~mm} / \mathrm{s}$. According to the above parameters and design process, select the model for the three-phase asynchronous motor. Y132S-8, speed is $710 \mathrm{r} / \mathrm{min}$, power is $2.2 \mathrm{kw}$, and maximum torque is $2.0 \mathrm{~N} \cdot \mathrm{M}$.

(2)Pneumatic actuator selection:

(1)The selection of the cylinder

Through access to "SMC pneumatic components selection guide", the inside diameter of cylinder is $16 \mathrm{~mm}$, and the air pressure is $0.5 \mathrm{MPa}$, the choice of the stroke of the cylinder is $150 \mathrm{~mm}$, and according to the manual, determine the standard cylinder series as type of CJ2, buffer form a gas buffer, and the installation is the form of earrings type. After checking calculation of the cylinder pressure, the model of the cylinder meets the requirements of design. The choice of lifting cylinder model is CJ2B16-150A.

The selection process of telescopic cylinder is similar with the lifting cylinder, but what should pay attention to is telescopic cylinder having a larger stroke, and it needs fully consider the bending moment applying to the cylinder guide, to guarantee the stability of cylinder. The final choice of cylinder type is MB1L32-450. The standard cylinder dimensions are as shown in Fig. 4.

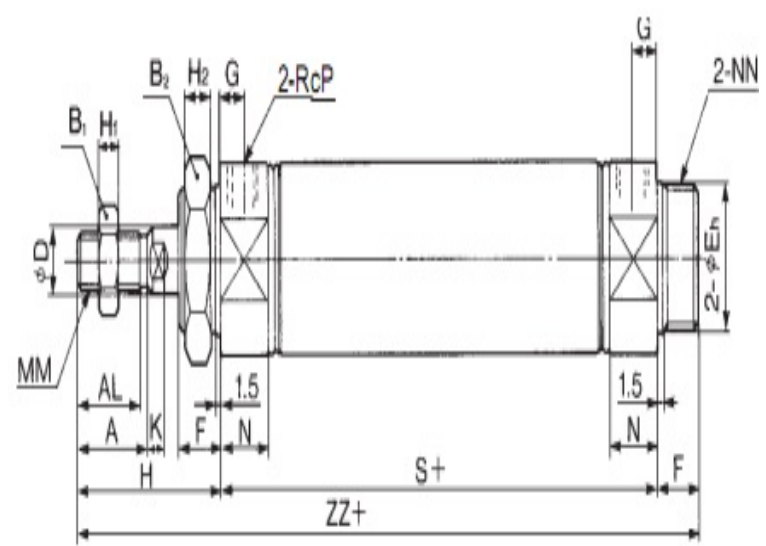

Fig. 4. Standard cylinder dimensions

(2) The choice of vacuum generator

The vacuum generator is a pneumatic device who used to produce vacuum, which has the advantages of simple structure, small volume, convenient installation and no moving mechanical parts, so it is widely used in modern industrial production. Principle of the structure is shown in Fig. 5.

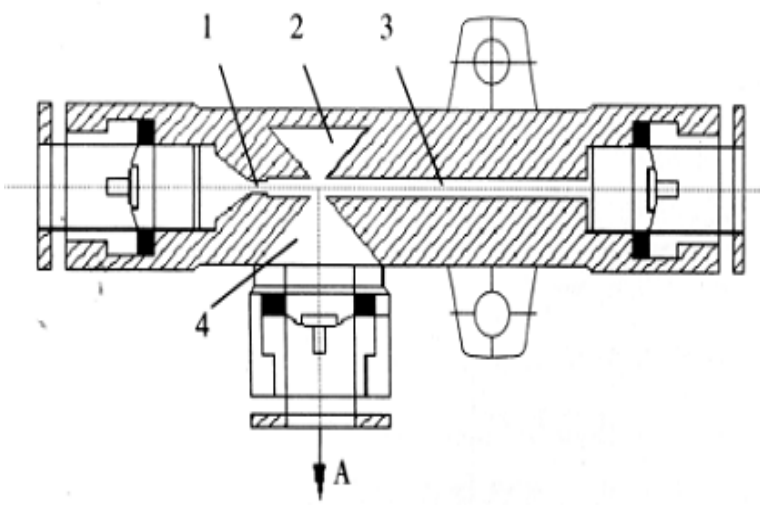

Fig. 5. Principle of the structure

According to the surface characteristics and structure characteristics of corrugated cardboard and it refers to the "SMC pneumatic components selection guide (third volumes)" and the selection basis is vacuum generator material. The choice of straight tube type vacuum generator model is ZU05L. Refer to the "SMC pneumatic components selection guide (third volumes)" and combined with the calculated numerical, the choice of models is ZP32UN: diameter 32, flat, polyurethane rubber material. The selected suction cup structure is shown in Fig. 6. 


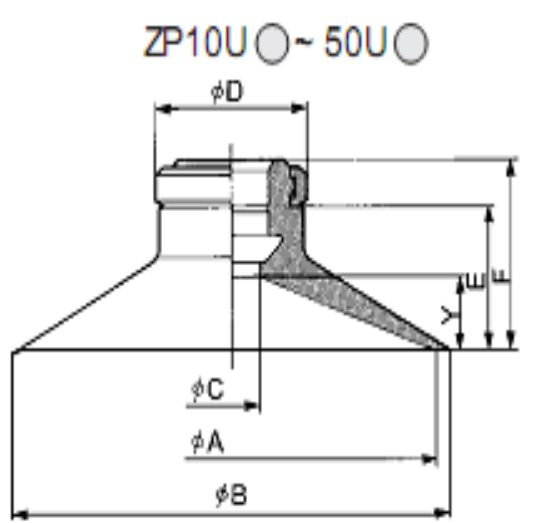

Fig. 6. Selected suction cup structure

3) The design of pneumatic system

The design of the control loop of the pneumatic manipulator is similar with traditional industrial manipulator, so in the design of the pneumatic loop take it as a reference, and make some improvements, including the design of vacuum adsorption loop.

C. The design of bending and holding unit.

The manipulator puts the cardboard on the front platform of the bending mechanism, and then puts it into the bending mechanism under the driving of conveying belt in the platform. The performance of bending mechanism will directly affect the whole manipulator system working efficiency, so the continuity, stability accuracy and operation mechanism for bending action completed is the focal point of design.

Structure design: The bending mechanism is composed of small gear, incomplete gear, reset spring, bearing, motor etc. According to the motion characteristics of the incomplete gear, meshing drive the rotation of the pinion, both of them are separated after driving the small gear to rotate for a certain angle, meanwhile, because of the action of the reset spring makes the bending (activities) board, quickly returned to in situ, namely, again with the main board in the same horizontal plane.

\section{CONCLUSIONS}

This paper is a design of nail box machine manipulator which is based on PLC control, from the overall design ideas to the concrete implementation of the program, all of them are met the design requirements. By improvement of the design of ladder diagram of PLC, succeed optimizing the control system, but also optimize part design agency, making the entire nail box machine manipulator system run more smoothly, automation transformation of automatic nail box machine on the basic of success.

\section{REFERENCES}

[1] Miura Hiromitsu, translated by Yang Xiaohui. The practical handbook for electromechanical integration [M]. Beijing: Science Press, 2007.

[2] Honda drought seedlings, Arai F. Translated by Zhou Yaokun. The design of handling machinery[M]. Beijing: Mechanical Industry Press.1978.

[3] Li Haolin, Fang Jian etc. Machine tool numerical control technology[M]. Beijing: Mechanical Industry Press.2007.5.

[4] Gong Zhonghua, Shi Jiancheng, Sun Yi. Mitsubishi FX/Q series of PLC application technology[M]. Beijing: People's Posts and Telecommunications Press, 2006.12

[5] Liu Liang. Advanced sensor and its application[M]. Beijing: Chemical Industry Press.2005.4.

[6] Ding Shifeng, Li Qingxiang. The design about a pneumatic feeding and discharging machine controlled by PLC[J]. Hydraulic and pneumatic 2012 fourth period. 